\title{
ON A RELATION TO TWO BASIC HILBERT-TYPE INTEGRAL INEQUALITIES
}

\author{
BICHENG YANG
}

\begin{abstract}
In this paper, by using the way of weight function and the technic of real analysis, a new integral inequality with some parameters and a best constant factor is given, which is a relation to two basic Hilbert-type integral inequalities. The equivalent form and the reverse forms are considered.
\end{abstract}

\section{Introduction}

If $f, g \geq 0,0<\int_{0}^{\infty} f^{2}(x) d x<\infty$ and $0<\int_{0}^{\infty} g^{2}(x) d x<\infty$ then we have the following basic Hilbert-type integral inequalities ${ }^{[1,2]}$ :

$$
\begin{gathered}
\int_{0}^{\infty} \int_{0}^{\infty} \frac{\ln (x / y) f(x) g(y)}{x-y} d x d y<\pi^{2}\left\{\int_{0}^{\infty} f^{2}(x) d x \int_{0}^{\infty} g^{2}(x) d x\right\}^{\frac{1}{2}} \\
\int_{0}^{\infty} \int_{0}^{\infty} \frac{f(x) g(y)}{|x-y|^{\lambda}} d x d y<2 B\left(1-\lambda, \frac{\lambda}{2}\right)\left\{\int_{0}^{\infty} x^{1-\lambda} f^{2}(x) d x \int_{0}^{\infty} x^{1-\lambda} g^{2}(x) d x\right\}^{\frac{1}{2}}
\end{gathered}
$$

where the constant factors $\pi^{2}$ and $2 B\left(1-\lambda, \frac{\lambda}{2}\right)(0<\lambda<1)$ are the best possible. The Beta function $B(u, v)$ is expressed by ${ }^{[3]}$ :

$$
B(u, v):=\int_{0}^{\infty} \frac{1}{(1+t)^{u+v}} t^{u-1} d t=\int_{0}^{1}(1-t)^{u-1} t^{v-1} d t \quad(u, v>0) .
$$

In recent years, by introducing two pairs of conjugate exponents and some parameters, some best extensions of them are established in $[4,5]$.

In this paper, by using the way of weight function and the technic of real analysis, a new integral inequality with the homogeneous kernel of $-\lambda$ degree

$$
\frac{|\ln (x / y)|^{\beta}}{|x-y|^{\lambda-\alpha}(\min \{x, y\})^{\alpha}}\left(\beta>-1, \lambda>0, \lambda-\beta-1<\alpha<\lambda \min \left\{\frac{1}{r}, \frac{1}{s}\right\}\right)
$$

Received April 28, 2008.

2000 Mathematics Subject Classification. 26D15.

Key words and phrases. Basic Hilbert-type integral inequality, Parameter, weight function. 
is given, which is a relation to the above two integral inequalities (1) and (2). The equivalent form and the reverse forms are considered. All the new inequalities possess the best constant factors.

\section{Some Lemmas}

We introduce the following Gamma function ${ }^{[3]}$ :

$$
\Gamma(s)=\int_{0}^{\infty} e^{-t} t^{s-1} d t(s>0) .
$$

Lemma 1. For $a, b>0$, it follows

$$
\int_{0}^{1} x^{a-1}(-\ln x)^{b-1} d x=\frac{1}{a^{b}} \Gamma(b)=\int_{1}^{\infty} y^{-a-1}(\ln y)^{b-1} d y .
$$

Proof. Setting $x=e^{-t / a}$ in the first integral of (5), by (4), we find the first equation of (5). Setting $y=1 / x$ in the first integral of (5), we obtain the last equation. The lemma is proved.

Lemma 2. If $r>1, \frac{1}{r}+\frac{1}{s}=1, \beta>-1, \lambda>0, \lambda-\beta-1<\alpha<\lambda \min \left\{\frac{1}{r}, \frac{1}{s}\right\}$, define the weight function as

$$
\varpi_{\lambda}(s, x):=x^{\frac{\lambda}{r}} \int_{0}^{\infty} \frac{|\ln (x / y)|^{\beta} y^{\frac{\lambda}{s}-1}}{|x-y|^{\lambda-\alpha}(\min \{x, y\})^{\alpha}} d y \quad(x \in(0, \infty)) .
$$

Then we have

$$
\varpi_{\lambda}(s, x)=k_{\lambda}(r):=\int_{0}^{\infty} \frac{|\ln u|^{\beta} u^{\frac{\lambda}{r}-1}}{|u-1|^{\lambda-\alpha}(\min \{u, 1\})^{\alpha}} d u,
$$

where $k_{\lambda}(r)$ is a positive number and

$$
k_{\lambda}(r)=\Gamma(\beta+1) \sum_{k=0}^{\infty}(-1)^{k}\left(\begin{array}{l}
\alpha-\lambda \\
k
\end{array}\right)\left[\frac{1}{\left(k+\frac{\lambda}{r}-\alpha\right)^{\beta+1}}+\frac{1}{\left(k+\frac{\lambda}{s}-\alpha\right)^{\beta+1}}\right] .
$$

Proof. Setting $u=x / y$ in (6), by simplification, we obtain (7). We find

$$
\begin{aligned}
0 & <k_{\lambda}(r)=\int_{0}^{1} \frac{(-\ln u)^{\beta} u^{\frac{\lambda}{r}-\alpha-1}}{(1-u)^{\lambda-\alpha}} d u+\int_{1}^{\infty} \frac{(\ln u)^{\beta} u^{\frac{\lambda}{r}-1}}{(u-1)^{\lambda-\alpha}} d u \\
& =\int_{0}^{1} \frac{(-\ln u)^{\beta} u^{\frac{\lambda}{r}-\alpha-1}}{(1-u)^{\lambda-\alpha}} d u+\int_{0}^{1} \frac{(-\ln v)^{\beta} v^{\frac{\lambda}{s}-\alpha-1}}{(1-v)^{\lambda-\alpha}} d v \\
& =\int_{0}^{1}\left[\frac{(-\ln u)^{\beta} u^{\delta}}{(1-u)^{\beta}}\right](1-u)^{(\alpha+\beta-\lambda+1)-1}\left[u^{\frac{\lambda}{r}-\alpha-\delta-1}+u^{\frac{\lambda}{s}-\alpha-\delta-1}\right] d u,
\end{aligned}
$$


where $0<\delta<\lambda \min \left\{\frac{1}{r}, \frac{1}{s}\right\}-\alpha$. Since $\frac{(-\ln u)^{\beta} u^{\delta}}{(1-u)^{\beta}} \rightarrow 0\left(u \rightarrow 0^{+}\right)$and $\frac{(-\ln u)^{\beta} u^{\delta}}{(1-u)^{\beta}} \rightarrow 1$ $\left(u \rightarrow 1^{-}\right)$, there exists $L>0$, such that $\frac{(-\ln u)^{\beta} u^{\delta}}{(1-u)^{\beta}} \leq L(u \in[0,1])$, then in view of (9) and (3), we have

$$
\begin{aligned}
0 & <k_{\lambda}(r) \leq L \int_{0}^{1}(1-u)^{(\alpha+\beta-\lambda+1)-1}\left[u^{\frac{\lambda}{r}-\alpha-\delta-1}+u^{\frac{\lambda}{s}-\alpha-\delta-1}\right] d u \\
& =L\left[B\left(\alpha+\beta-\lambda+1, \frac{\lambda}{r}-\alpha-\delta\right)+B\left(\alpha+\beta-\lambda+1, \frac{\lambda}{s}-\alpha-\delta\right)\right],
\end{aligned}
$$

and $k_{\lambda}(r)$ is a positive number. Still by (9), using the property of power series, we find

$$
\begin{aligned}
k_{\lambda}(r) & =\int_{0}^{1}(1-u)^{\alpha-\lambda}(-\ln u)^{\beta}\left[u^{\frac{\lambda}{r}-\alpha-1}+u^{\frac{\lambda}{s}-\alpha-1}\right] d u \\
& =\int_{0}^{1} \sum_{k=0}^{\infty}(-1)^{k}\left(\begin{array}{l}
\alpha-\lambda \\
k
\end{array}\right)(-\ln u)^{\beta}\left[u^{\frac{\lambda}{r}-\alpha+k-1}+u^{\frac{\lambda}{s}-\alpha+k-1}\right] d u \\
& =\sum_{k=0}^{\infty}(-1)^{k}\left(\begin{array}{l}
\alpha-\lambda \\
k
\end{array}\right) \int_{0}^{1}(-\ln u)^{(\beta+1)-1}\left[u^{\frac{\lambda}{r}-\alpha+k-1}+u^{\frac{\lambda}{s}-\alpha+k-1}\right] d u .
\end{aligned}
$$

Then in view of (5), we have (8). The lemma is proved.

Lemma 3. If $p>0(p \neq 1), r>1, \frac{1}{p}+\frac{1}{q}=1, \frac{1}{r}+\frac{1}{s}=1, \beta>-1, \lambda>0$, $\lambda-\beta-1<\alpha<\lambda \min \left\{\frac{1}{r}, \frac{1}{s}\right\}, n \in N, n>\frac{r}{|q| \lambda}$, then for $n \rightarrow \infty$, we have

$$
I_{n}:=\frac{1}{n} \int_{1}^{\infty} \int_{1}^{\infty} \frac{\left|\ln \left(\frac{x}{y}\right)\right|^{\beta} x^{\frac{\lambda}{r}-\frac{1}{n p}-1} y^{\frac{\lambda}{s}-\frac{1}{n q}-1}}{|x-y|^{\lambda-\alpha}(\min \{x, y\})^{\alpha}} d x d y=k_{\lambda}(r)+o(1)
$$

Proof. Setting $u=y / x$, by Fubini's theorem ${ }^{[6]}$, we obtain

$$
\begin{aligned}
I_{n} & =\frac{1}{n} \int_{1}^{\infty}\left[\int_{1}^{\infty} \frac{\left|\ln \left(\frac{x}{y}\right)\right|^{\beta} x^{\frac{\lambda}{r}-\frac{1}{n p}-1} y^{\frac{\lambda}{s}-\frac{1}{n q}-1}}{|x-y|^{\lambda-\alpha}(\min \{x, y\})^{\alpha}} d x\right] d y \\
& =\frac{1}{n} \int_{1}^{\infty} y^{-\frac{1}{n}-1}\left[\int_{0}^{y} \frac{|\ln u|^{\beta} u^{\frac{\lambda}{s}+\frac{1}{n p}-1}}{|1-u|^{\lambda-\alpha}(\min \{1, u\})^{\alpha}} d u\right] d y \\
& =\frac{1}{n} \int_{1}^{\infty} y^{-\frac{1}{n}-1}\left[\int_{0}^{1} \frac{(-\ln u)^{\beta} u^{\frac{\lambda}{s}+\frac{1}{n p}-1}}{(1-u)^{\lambda-\alpha} u^{\alpha}} d u+\int_{1}^{y} \frac{(\ln u)^{\beta} u^{\frac{\lambda}{s}+\frac{1}{n p}-1}}{(u-1)^{\lambda-\alpha}} d u\right] d y \\
& =\int_{0}^{1} \frac{(-\ln u)^{\beta} u^{\frac{\lambda}{s}+\frac{1}{n p}-1}}{(1-u)^{\lambda-\alpha} u^{\alpha}} d u+\frac{1}{n} \int_{1}^{\infty} y^{-\frac{1}{n}-1}\left[\int_{1}^{y} \frac{(\ln u)^{\beta} u^{\frac{\lambda}{s}+\frac{1}{n p}-1}}{(u-1)^{\lambda-\alpha}} d u\right] d y \\
& =\int_{0}^{1} \frac{(-\ln u)^{\beta} u^{\frac{\lambda}{s}+\frac{1}{n p}-1}}{(1-u)^{\lambda-\alpha} u^{\alpha}} d u+\frac{1}{n} \int_{1}^{\infty}\left(\int_{u}^{\infty} y^{-\frac{1}{n}-1} d y\right) \frac{(\ln u)^{\beta} u^{\frac{\lambda}{s}+\frac{1}{n p}-1}}{(u-1)^{\lambda-\alpha}} d u
\end{aligned}
$$




$$
=\int_{0}^{1} \frac{(-\ln u)^{\beta} u^{\frac{\lambda}{s}-\alpha+\frac{1}{n p}-1}}{(1-u)^{\lambda-\alpha}} d u+\int_{1}^{\infty} \frac{(\ln u)^{\beta} u^{\frac{\lambda}{s}-\frac{1}{n q}-1}}{(u-1)^{\lambda-\alpha}} d u
$$

(i) If $p>0(p \neq 1)$ and $q>0$, by Levi's theorem $[6]$, we find

$$
\begin{aligned}
\int_{0}^{1} \frac{(-\ln u)^{\beta} u^{\frac{\lambda}{s}-\alpha+\frac{1}{n p}-1}}{(1-u)^{\lambda-\alpha}} d u & =\int_{0}^{1} \frac{(-\ln u)^{\beta} u^{\frac{\lambda}{s}-\alpha-1}}{(1-u)^{\lambda-\alpha}} d u+o_{1}(1), \\
\int_{1}^{\infty} \frac{(\ln u)^{\beta} u^{\frac{\lambda}{s}-\frac{1}{n q}-1}}{(u-1)^{\lambda-\alpha}} d u & =\int_{1}^{\infty} \frac{(\ln u)^{\beta} u^{\frac{\lambda}{s}-1}}{(u-1)^{\lambda-\alpha}} d u+o_{2}(1) \quad(n \rightarrow \infty) ;
\end{aligned}
$$

(ii) if $q<0$, setting $n_{0} \in N, n_{0}>\frac{r}{|q| \lambda}, \frac{1}{s^{\prime}}=\frac{1}{s}-\frac{1}{n_{0} q \lambda}, \frac{1}{r^{\prime}}=\frac{1}{r}+\frac{1}{n_{0} q \lambda}$, then for $n \geq n_{0}$, we have

$$
\int_{1}^{\infty} \frac{(\ln u)^{\beta} u^{\frac{\lambda}{s}-\frac{1}{n^{2}}-1}}{(u-1)^{\lambda-\alpha} u^{\alpha}} d u \leq \int_{1}^{\infty} \frac{(\ln u)^{\beta} u^{\frac{\lambda}{s}-\frac{1}{n_{0} q}-1}}{(u-1)^{\lambda-\alpha} u^{\alpha}} d u \leq k_{\lambda}\left(r^{\prime}\right)<\infty,
$$

and by Lebesgue's control convergence theorem ${ }^{[6]}$, we have

$$
\int_{1}^{\infty} \frac{(\ln u)^{\beta} u^{\frac{\lambda}{s}-\frac{1}{n q}-1}}{(u-1)^{\lambda-\alpha}} d u=\int_{1}^{\infty} \frac{(\ln u)^{\beta} u^{\frac{\lambda}{s}-1}}{(u-1)^{\lambda-\alpha}} d u+o_{3}(1) \quad(n \rightarrow \infty) .
$$

In view of the above results and (11), we have (10). The lemma is proved.

\section{Main Results}

Theorem 1. Assume that $p>0(p \neq 1), r>1, \frac{1}{p}+\frac{1}{q}=1, \frac{1}{r}+\frac{1}{s}=1, \beta>-1$, $\lambda>0, \lambda-\beta-1<\alpha<\lambda \min \left\{\frac{1}{r}, \frac{1}{s}\right\}, \phi(x)=x^{p\left(1-\frac{\lambda}{r}\right)-1}, \psi(x)=x^{q\left(1-\frac{\lambda}{s}\right)-1}(x \in(0, \infty))$, and $f, g \geq 0,0<\|f\|_{p, \phi}=\left\{\int_{0}^{\infty} x^{p\left(1-\frac{\lambda}{r}\right)-1} f^{p}(x) d x\right\}^{\frac{1}{p}}<\infty, 0<\|g\|_{q, \psi}<\infty$. (i) For $p>1$, we have the following inequality:

$$
I:=\int_{0}^{\infty} \int_{0}^{\infty} \frac{|\ln (x / y)|^{\beta} f(x) g(y)}{|x-y|^{\lambda-\alpha}(\min \{x, y\})^{\alpha}} d x d y<k_{\lambda}(r)\|f\|_{p, \phi}\|g\|_{q, \psi},
$$

where the constant factor $k_{\lambda}(r)$ expressed by (8) is the best possible; (ii) for $0<p<1$, we have the reverse of (12) with the best constant factor $k_{\lambda}(r)$.

Proof. (i) For $p>1$, by Hölder's inequality with weight ${ }^{[7]}$, in view of (6), we find

$$
\begin{aligned}
I & =\int_{0}^{\infty} \int_{0}^{\infty} \frac{|\ln (x / y)|^{\beta}}{|x-y|^{\lambda-\alpha}(\min \{x, y\})^{\alpha}}\left[\frac{x^{\left(1-\frac{\lambda}{r}\right) / q}}{y^{\left(1-\frac{\lambda}{s}\right) / p}} f(x)\right]\left[\frac{y^{\left(1-\frac{\lambda}{s}\right) / p}}{x^{\left(1-\frac{\lambda}{r}\right) / q}} g(y)\right] d x d y \\
& \leq\left\{\int_{0}^{\infty} \int_{0}^{\infty} \frac{|\ln (x / y)|^{\beta}}{|x-y|^{\lambda-\alpha}(\min \{x, y\})^{\alpha}} \cdot \frac{x^{\left(1-\frac{\lambda}{r}\right)(p-1)}}{y^{1-\frac{\lambda}{s}}} f^{p}(x) d x d y\right\}^{\frac{1}{p}}
\end{aligned}
$$




$$
\begin{aligned}
& \times\left\{\int_{0}^{\infty} \int_{0}^{\infty} \frac{|\ln (x / y)|^{\beta}}{|x-y|^{\lambda-\alpha}(\min \{x, y\})^{\alpha}} \cdot \frac{y^{\left(1-\frac{\lambda}{s}\right)(q-1)}}{x^{1-\frac{\lambda}{r}}} g^{q}(y) d x d y\right\}^{\frac{1}{q}} \\
= & \left\{\int_{0}^{\infty} \varpi_{\lambda}(s, x) \phi(x) f^{p}(x) d x\right\}^{\frac{1}{p}}\left\{\int_{0}^{\infty} \varpi_{\lambda}(r, y) \psi(y) g^{q}(y) d y\right\}^{\frac{1}{q}} .
\end{aligned}
$$

We conform that inequality (13) keeps the strict form. Otherwise, there exist constants $A$ and $B$, such that they are not all zero and ${ }^{[7]}$

$$
A \frac{x^{\left(1-\frac{\lambda}{r}\right)(p-1)}}{y^{1-\frac{\lambda}{s}}} f^{p}(x)=B \frac{y^{\left(1-\frac{\lambda}{s}\right)(q-1)}}{x^{1-\frac{\lambda}{r}}} g^{q}(y) \text { a. e. in }(0, \infty) \times(0, \infty) .
$$

It follows $A x^{p\left(1-\frac{\lambda}{r}\right)} f^{p}(x)=B y^{q\left(1-\frac{\lambda}{s}\right)} g^{q}(y)$ a.e.in $(0, \infty) \times(0, \infty)$. Assuming that $A \neq 0$, there exists $y>0$, such that $x^{p\left(1-\frac{\lambda}{r}\right)-1} f^{p}(x)=\left[B y^{q\left(1-\frac{\lambda}{s}\right)} g^{q}(y)\right] \frac{1}{A x}$ a.e. in $x \in(0, \infty)$. This contradicts the fact that $0<\|f\|_{p, \phi}<\infty$. Then inequality (12) is valid by using (7) and (8).

For $n \in N, n>\frac{r}{|q| \lambda}$, setting $f_{n}, g_{n}$ as

$$
f_{n}(x):=\left\{\begin{array}{l}
0, \quad 0<x \leq 1 \\
x^{\frac{\lambda}{r}-\frac{1}{n p}-1}, \quad x>1
\end{array}, \quad g_{n}(x):=\left\{\begin{array}{l}
0, \quad 0<x \leq 1 \\
x^{\frac{\lambda}{r}-\frac{1}{n q}-1}, \quad x>1
\end{array},\right.\right.
$$

if there exists a constant factor $0<k \leq k_{\lambda}(r)$, such that (12) is valid if we replace $k_{\lambda}(r)$ by $k$, then by (10), we have

$$
\begin{aligned}
k_{\lambda}(r)+o(1) & =I_{n}=\frac{1}{n} \int_{0}^{\infty} \int_{0}^{\infty} \frac{\left|\ln \left(\frac{x}{y}\right)\right|^{\beta} f_{n}(x) g_{n}(y)}{|x-y|^{\lambda-\alpha}(\min \{x, y\})^{\alpha}} d x d y \\
& <\frac{1}{n} k\left\|f_{n}\right\|_{p, \phi}\left\|g_{n}\right\|_{q, \psi}=k,
\end{aligned}
$$

and $k_{\lambda}(r) \leq k(n \rightarrow \infty)$. Hence $k=k_{\lambda}(r)$ is the best constant factor of (12).

(ii) For $0<p<1$, by the reverse Hölder's inequality with weight ${ }^{[7]}$, in view of (6), we find the reverse of (13), which still keeps the strict form. Then by (7) and (8), we have the reverse of (12). By (10) and the same way as the above mention, we can show that the constant factor in the reverse of (12) is still the best possible. The theorem is proved.

Theorem 2. Assume that $p>0(p \neq 1), r>1, \frac{1}{p}+\frac{1}{q}=1, \frac{1}{r}+\frac{1}{s}=1, \beta>-1$, $\lambda>0, \lambda-\beta-1<\alpha<\lambda \min \left\{\frac{1}{r}, \frac{1}{s}\right\}, \phi(x)=x^{p\left(1-\frac{\lambda}{r}\right)-1}, \psi(x)=x^{q\left(1-\frac{\lambda}{s}\right)-1}(x \in(0, \infty))$, and $f \geq 0,0<\|f\|_{p, \phi}<\infty$. (i) For $p>1$, we have the following inequality equivalent to (12):

$$
J:=\int_{0}^{\infty} y^{\frac{p \lambda}{s}-1}\left[\int_{0}^{\infty} \frac{|\ln (x / y)|^{\beta} f(x)}{|x-y|^{\lambda-\alpha}(\min \{x, y\})^{\alpha}} d x\right]^{p} d y<k_{\lambda}^{p}(r)\|f\|_{p, \phi}^{p},
$$


where the constant factor $k_{\lambda}^{p}(r)$ is the best possible; (ii) for $0<p<1$, we have the reverse of (14), which is equivalent to the reverse of (12) with the best constant factor $k_{\lambda}^{p}(r)$.

Proof. (i) For $p>1, x>0$, setting a bounded measurable function as

$$
[f(x)]_{n}:=\min \{f(x), n\}=\left\{\begin{array}{l}
f(x), \text { for } f(x)<n \\
n, \text { for } f(x) \geq n
\end{array},\right.
$$

since $\|f\|_{p, \phi}>0$, there exists $n_{0} \in N$, such that $\int_{\frac{1}{n}}^{n} \phi(x)[f(x)]_{n}^{p} d x>0\left(n \geq n_{0}\right)$, Setting $\widetilde{g}_{n}(y)\left(y \in\left(\frac{1}{n}, n\right) ; n \geq n_{0}\right)$ as

$$
\widetilde{g}_{n}(y):=y^{\frac{p \lambda}{s}-1}\left[\int_{\frac{1}{n}}^{n} \frac{|\ln (x / y)|^{\beta}}{|x-y|^{\lambda-\alpha}(\min \{x, y\})^{\alpha}}[f(x)]_{n} d x\right]^{p-1},
$$

then by (12), we find

$$
\begin{aligned}
0 & <\int_{\frac{1}{n}}^{n} \psi(y) \widetilde{g}_{n}^{q}(y) d y=\int_{\frac{1}{n}}^{n} y^{\frac{p \lambda}{s}-1}\left[\int_{\frac{1}{n}}^{n} \frac{|\ln (x / y)|^{\beta}[f(x)]_{n} d x}{|x-y|^{\lambda-\alpha}(\min \{x, y\})^{\alpha}}\right]^{p} d y \\
& =\int_{\frac{1}{n}}^{n} \int_{\frac{1}{n}}^{n} \frac{|\ln (x / y)|^{\beta}[f(x)]_{n} \widetilde{g}_{n}(y)}{|x-y|^{\lambda-\alpha}(\max \{x, y\})^{\alpha}} d x d y \\
& <k_{\lambda}(r)\left\{\int_{\frac{1}{n}}^{n} \phi(x)[f(x)]_{n}^{p} d x\right\}^{\frac{1}{p}}\left\{\int_{\frac{1}{n}}^{n} \psi(y) \widetilde{g}_{n}^{q}(y) d y\right\}^{\frac{1}{q}}<\infty ; \\
0 & <\int_{\frac{1}{n}}^{n} \psi(y) \widetilde{g}_{n}^{q}(y) d y<k_{\lambda}^{p}(r) \int_{0}^{\infty} \phi(x) f^{p}(x) d x<\infty .
\end{aligned}
$$

It follows $0<\|g\|_{q, \psi}<\infty$. For $n \rightarrow \infty$, by (12), both (16) and (17) still keep the forms of strict inequality. Hence we have (14). On the other-hand, suppose (14) is valid. By Hölder's inequality, we have

$$
I=\int_{0}^{\infty}\left[y^{\frac{-1}{p}+\frac{\lambda}{s}} \int_{0}^{\infty} \frac{|\ln (x / y)|^{\beta} f(x) d x}{|x-y|^{\lambda-\alpha}(\min \{x, y\})^{\alpha}}\right]\left[y^{\frac{1}{p}-\frac{\lambda}{s}} g(y)\right] d y \leq J^{\frac{1}{p}}\|g\|_{q, \psi} .
$$

In view of (14), we have (12), which is equivalent to (14). We conform that the constant factor in (14) is the best possible. Otherwise, we may get a contradiction by (18) that the constant factor in (12) is not the best possible.

(ii) For $0<p<1$, since $\|f\|_{p, \phi}>0$, we conform that $J>0$. If $J=\infty$, then the reverse of (14) is naturally valid. Suppose $0<J<\infty$. Setting

$$
g(y):=y^{\frac{p \lambda}{s}-1}\left(\int_{0}^{\infty} \frac{|\ln (x / y)|^{\beta}}{|x-y|^{\lambda-\alpha}(\max \{x, y\})^{\alpha}} f(x) d x\right)^{p-1},
$$

by the reverse of (12), we obtain

$$
\infty>\|g\|_{q, \psi}^{q}=J=I>k_{\lambda}(r)\|f\|_{p, \phi}\|g\|_{q, \psi}>0
$$




$$
J^{\frac{1}{p}}=\|g\|_{q, \psi}^{q-1}>k_{\lambda}(r)\|f\|_{p, \phi} .
$$

Hence we have the reverse of (14). On the other-hand, suppose the reverse of (14) is valid. By the reverse Hölder's inequality, we can get the reverse of (18). Hence in view of the reverse of (14), we obtain the reverse of (12), which is equivalent to the reverse of (14). We conform that the constant factor in the reverse of (14) is the best possible. Otherwise, we may get a contradiction by the reverse of (18) that the constant factor in the reverse of (12) is not the best possible. The theorem is proved.

Remarks. For $p=r=2$ in (12), setting $\alpha=\beta=0$, we have (2); setting $\alpha=0$, $\beta=\lambda=1$, we have (1). Hence inequality (12) is a relation to (1) and (2) with the best constant factor.

\section{References}

[1] G. H. Hardy, J. E. Littlewood and G. Polya, Inequalities, Cambridge Univ. Press, Cambridge, 1952.

[2] Bicheng Yang, A new Hilbert-type integral inequality and its generalization, Journal of Jilin University, 43(5)(2005), 580-584.

[3] Zhuxi Wang and Dunren Guo, Introduction to Special Functions, Science Press, Beijing, 1979.

[4] Wuyi Zhong and Bicheng Yang, A best extension of Hilbert inequality involving several parameters, Journal of Jinan University (Natural Science), 28(2007), 20-23.

[5] Wuyi Zhong and Bicheng Yang, A best extension of new Hilbert's type integral inequality with some parameters, Journal of Jiangxi Normal University (Natural Science), 31(2007), 410-414.

[6] Jichang Kuang, Introduction to Real Analysis, Hunan Education Press, Changsha, 1996.

[7] Jichang Kuang, Applied Inequalities, Shangdong Science Press, Jinan, 2004.

Department of Mathematics, Guangdong Education Institute, Guangzhou, Guangdong 510303, P. R. China.

E-mail: bcyang@pub.guangzhou.gd.cn 